\title{
ПРОБЛЕМА ВЫБОРА ЛУЧШЕГО ТЕХНИЧЕСКОГО РЕШЕНИЯ ДЛЯ ОБЕСПЕЧЕНИЯ ВАКУУМ-ВЫПАРНОЙ УСТАНОВКИ ПОДВОДОМ ТЕПЛА
}

Смирнов Г.Ф., Д.т.н., Зыков А.В., канд. техн. наук, Резниченко Д.Н.

Одесская национальная академия пищевых технологий, ул. Канатная 112, Одесса, Украина

Метою досліджень була розробка методики визначення показників енергоефективності різних схем енергозабезпечення ВВУ. В результаті проведено порівняння одноступеневої ВВУ і ВВУ $з$ тепловим насосом. Для ВВУ $з$ тепловим насосом визначені режимні параметри які забезпечують мінімум обраного критерію.

Библ.10. рис. 2.
Целью исследований являлась разработка методики определения показателей энергоэффективности различных схем энергообеспечения ВВУ. В результате проведено сравнение одноступенчатой ВВУ и BВУ с тепловым насосом. Для ВВУ c тепловым насосом определены режимные параметры обеспечивающие минимум выбранного критерия.

\begin{abstract}
The goal of research was to develop a methodology for determining the energy efficiency of various schemes of Vacuum Evaporators (VE) energy supply. As a result, the comparison of singleeffect VE and VE with heat pump (HPVE) was done. For HPVE the operating parameters are defined to ensure a minimum of the selected criteria.
\end{abstract}

Ключевые слова: вакуум-выпарной аппарат, тепловой насос, оптимизация.

\section{Введение.}

Одна из распространенных форм организации технологического процесса в пищевой, химической, биотехнической и др. областей промышленности связана с подводом тепла низкого температурного потенциала. При этом решается вопрос об источнике тепла. При обычном подходе используется греющий пар, получаемый от котельной. Иногда этот пар может быть получен как часть отбираемого пара из отборов паровой турбины [1]. Тогда, чем ниже давление и температура отбора, тем выше получаемая от него энергия. Но понижение температуры греющего пара, при требуемом по технологии уровне, приводит к возрастанию поверхности теплообмена, на которой должен кипеть продукт. Это означает необходимость выбора оптимума этого параметра. Решение этой проблемы, по существу, задача оптимизации ВВУ при её стационарном режиме.

Первый шаг связан с определением функции цели. Естественно, принимать в качестве такой величины суммарные затраты в денежном выражении. В этом случае, как правило, они определяются как сумма затрат, в которую входят: a) Затраты эксплуатационные, включая затраты на подачу энергии и тепла; обслуживание работы ВВУ и т.п. б) Затраты капитальные, связанные с стоимостью оборудования, приборов, необходимых помещений и пр.

Совремённые тенденции, обусловленные энергетическими проблемами, приводят к необходимости, учитывать задачи энерго - ресурсосбережения $[2,3]$. Традиционным путем повышения энергетической эффективности процесса при сохранении качества концентрируемого продукта является использование многоступенчатых BВУ $[4,5]$. Также для снижения затрат энергии на процесс используется и энергия отводимого вторичного пара. Существуют схемные решения организации работы ВВУ, основанные на применении разных вариантов устройств по сжатию вторичного пара и возврата его в качестве источника тепла, такие как использование эжекторов $[6,7]$, применение компрессоров или газодувок [8], и разнообразные их вариации. Эффективность такого пути решения проблемы энергосбережения сильно зависит от экономических показателей этих устройств: в частности к.П.д. (этот параметр достаточно мал для эжекторов, в первую очередь,). В не меньшей мере, это зависит от 
уровня отработки этих устройств и данных по их стоимости.

В последние годы оказались популярными и перспективными схемы обеспечения работы ВВУ на основе использования тепловых насосов (ТНУ) в качестве источников подвода тепла $[9,10]$, которые, при соблюдении требований технологии, могут решать и задачи отвода тепла, возникающие в ВВУ, связанные с конденсацией вторичного пара, образующегося как побочный результат при выпаривании. Таким образом, возникает задача объективного сравнения двух схемных решений технологии ВВУ: обычной, с греющим паром в качестве основного источника тепла, и с применением ТНУ. Представляется, что в этом отношении, применение ТНУ имеет преимущество. Поэтому, в данной работе авторами исследовалась проблема объективного сравнения 2-х схем ВВУ: 1. Использующих греющий пар; 2. Использующих ТНУ, в качестве источника энергии. В качестве конкретного примера рассматривалась одноступенчатая ВВУ для концентрирования водных растворов. Такие ВВУ весьма распространены в пищевых технологиях. Заметим также, что они же применимы при решении экологических проблем (очистка сточных вод; промышленных стоков и т.д.); в химических технологиях при пониженном уровне температур и др.

\section{Процедура и методика расчёта ВВУ с гре- ющцим паром в качестве источника тепла. Тоже при использовании ТНУ как источника и стока тепла в ВВУ}

Целью расчета является определение конструктивных параметров установки и определение затрат энергии на проведение процесса. Затраты энергии складываются из затрат на подогрев продукта до температуры кипения, на испарение растворителя и компенсацию потерь энергии в окружающую среду. Основным же конструктивным параметром является площадь теплопередающей поверхности, которая определяется из уравнения теплопередачи. Следует отметить, что при использовании ТНУ как источника и стока теплоты, конденсация вторичного пара осуществляется в поверхностном теплообменнике и его поверхность также должна быть определена при расчете. Набор исходных данных, рас- чётных формул и общий порядок расчёта имеет следующий вид:

1.Производительность по начальному продукту $G_{n}$ в кг/час или в кг/сек.

2. Начальное содержание сухих веществ в кг/ кг $a$;

3. Конечное содержание сухих веществ в кг/ кг $a_{k}$;

4. Давление греющего пара в МПа $P_{g r}$, температура $t_{g r}$ и её диапазон.

5. Температура и давление вторичного пара в градусах С и в МПа $P_{v t}$.

6. Температура выпаривания в градусах $\mathrm{C} t_{v t}$. и диапазон её изменения.

7. Начальная температура продукта $t_{n}$ в градусах C.

8. Степень сухости греющего пара или его энтальпия.

9. Материал поверхностей теплообменников и его физические свойства.

10. Тип топлива его теплотворная способность и стоимость его единицы.

11. Стоимости основного оборудования и сроки его эксплуатации.

12. Соотношения для определения теплофизических свойств теплоносителя.

13. Соотношения для определения теплофизических свойств продукта.

В последних важными являются соотношения для расчёта основных температурных депрессий конечного состояния продукта: физико-химической (ФХТД), гидростатической, гидродинамической. Для первой необходима информация, учитывающая реальные свойства продукта на выходе; для второй нужны данные по конструкции парогенератора; третью принимают, а затем уточняют после завершения основных расчётов. Ниже дана формула для расчёта ФХТД при выпаривании соков:

$\Delta \mathrm{ph}=0.38 \cdot \exp \left(0.05+0.045 \cdot \mathrm{a}_{\mathrm{k}}\right)$,

здесь $\Delta \mathrm{ph} ; \mathrm{a}_{\mathrm{k}}$ ФХТД и концентрация на выходе продукта, соответственно. Для гидростатической депрессии рекомендуется следующая схема расчёта оптимальной высоты продукта в трубках парогенератора;

$l_{\text {opt }}=\left[0.26+0.0014 \cdot \frac{\left(\rho_{p r}-\rho_{g r k}(t v t)\right.}{\mathrm{kg} \cdot \mathrm{m}^{-3}}\right] \cdot \mathrm{m}$, 
здесь: $l_{o p t} ; \rho_{g r k}(t v t) ; \rho_{p r}$ - оптимальная высота уровня кипящего продукта в трубках парогенератора; его плотность на выходе и на входе, соответственно. Далее, среднее давление в аппарате:

$P_{c p}=P_{v t}+0.5 \cdot$ lopt $\cdot \rho_{p r} \cdot g$.

Масса выпаренной влаги определяется:

$W_{v t}=G_{n} \cdot\left(1-a_{n} / a_{k}\right)$.

здесь $W_{v t}$ и есть массовый расход в кг/сек или в кг/час. Тепловая мощность необходимая для обеспечения технологии $Q_{0}$ состоит: 1 мощности для нагрева от начальной температуры продукта до температуры насыщения $Q_{1}$ при его парообразовании; 2. мощности для самого процесса выпаривания $Q_{2}$; 3. потерь тепла в окружающую среду Qop. Формулы для определения этих слагаемых имеют следующий вид:

$Q_{1}=G_{n} \cdot C_{p} \cdot\left(t_{s}-t_{n}\right)$

$Q_{2}=W_{v t} \cdot r_{v t}$

$Q_{o p}=0.05 *\left(Q_{1}+Q_{2}\right)$;

$Q_{0}=Q_{1}+Q_{2}+Q_{o p}$

Температурный напор в парогенераторе (независимая переменная) определяется как:

$\Delta t_{i}=t_{g r i}-t_{S}$,

здесь $\Delta t_{i}, t_{g r i}, t_{S}-$ i ый температурный напор; і я температура нагрева; температура насыщения при выпаривании, соответственно. Следующими действиями будут расчёты теплопередачи в греющей камере парогенератора. Плотность теплового потока в ней определяется по данным расчётов теплоотдачи с сторон кипения и конденсации. С стороны кипения продукта коэффициент теплоотдачи можно рассчитать по следующей форму-

ле: $\propto 2=(\lambda 1 / \delta 1) \cdot 3.25 \cdot 10^{-4} \cdot\left(q 1 f \cdot \frac{\delta 1}{r 1 \cdot \rho 2 \cdot \lambda 1} \cdot\right.$

$\cdot C 1 \cdot \rho 1)^{0.6} \cdot\left(g \cdot \frac{\delta 1^{3}}{v 1^{2}}\right)^{0.125} \cdot\left(P 1 \cdot \frac{\delta 1}{\sigma 1}\right)^{0.7}$.

Здесь:

$\delta 1=\sqrt{\frac{\sigma 1}{(\rho 1-\rho 2) \cdot g}} ;$

$q 1 f=\alpha 2 * \Delta t 2$;

$\Delta t 2=t_{\text {gri2 }}-t_{S \text {, }}$

прочие величины связаны с теплофизическими свойствами продукта; это: $\lambda 1 ; r 1 ; \rho 2 ; \rho 1 ; \nu 1 ; \sigma 1 ; C 1$; $P 1$ - коэффициент теплопроводности жидкого продукта; теплота его фазового перехода; плотности пара и жидкости; коэффициенты кинематической вязкости и поверхностного натяжения жидкого продукта; его удельная теплоёмкость и давление насыщения, соответственно. Для расчёта коэффициента теплоотдачи при конденсации внутри трубок парогенератора используем известную зависимость для полной конденсации быстродвижущегося пара внутри гладкого канала:

$\frac{\propto 1 \cdot d}{\lambda 1}=C 10 \cdot \operatorname{Re} 1^{0.8} \cdot \operatorname{Pr} 1^{0.43} \cdot\left[1+X_{\mathrm{cp}} \cdot(\rho 1 / \rho 2-1)\right]^{0.5}$.

Здесь: $\alpha 1 ; \mathrm{d} 1 ; \mathrm{C} 10 ; \operatorname{Re} 1 ; \operatorname{Pr} 1 ; \mathrm{X}_{c p} ; \rho 1 ; \rho 2 ; \lambda 1$ - средний по поверхности канала коэффициент теплоотдачи при конденсации внутри быстродвижущегося пара с средним массовым расходным паросодержанием, равным $\mathrm{X}_{c p}$; внутренний диаметр канала; полуэмпирическая постоянная; число Рейнольдса для плёночной конденсации; число Прандтля для конденсата; среднее массовое расходное паросодержание; плотности конденсата и пара; коэффициент теплопроводности конденсата, соответственно. В (14)

$\operatorname{Re} 1=\frac{q \cdot d 1}{\mathrm{r} 1 \cdot \mu 1}$,

тут $q$ - средняя по внутренней поверхности канала плотность теплового потока; r1; $\mu 1$ - теплота фазового перехода и динамическая вязкость конденсата, соответственно. Термическое сопротивление с стороны кипящего продукта помимо термического сопротивления процесса, включает сумму термических сопротивлений металлической стенки и загрязнений, т.е.

$R_{\Sigma}=\left(\frac{\delta_{w}}{\lambda_{w}}+R_{\text {заг }}\right)$,

тут $R_{\Sigma} ; \delta_{w} ; \lambda_{w} ; R_{\text {заг }}$ - сумма 2 -х этих сопротивлений; толщина стенки и её коэффициент теплопроводности, термическое сопротивление загрязнений, соответственно. Последнюю величину нужно принимать на основе рекомендаций из эксплуатационного опыта. Таким образом, расчёт интенсивности теплопередачи через стенку парогенератора при конденсации греющего пара внутри трубы и кипении продукта на внешней её поверхности приводит к системе трансцендентных уравнений или к системе из степенных уравнений вида:

$\alpha 2:=\mathrm{Z} 11 \cdot \Delta \mathrm{t} 2^{1.5}$, 
здесь $\alpha 2, \Delta t_{2}, q$ - коэффициент теплоотдачи с стороны кипящего продукта; температурный напор поверхность кипения - объём кипящей среды; плотность теплового потока, соответственно. Параметр Z11 - есть комплекс теплофизических свойств следующих из эмпирической формулы для расчёта интенсивности процесса кипения. Этот параметр имеет вид:

$$
Z 11=\delta 1^{1.675} \cdot \lambda 1 \cdot\left(C 1 \cdot \frac{\rho 1}{\rho 2} \cdot \frac{1}{r 1}\right)^{1.5} \cdot\left(\frac{P 1}{\sigma 1}\right)^{1.75} \cdot(g)^{0.3125} \cdot(v 1)^{-0.625},(1
$$

следует иметь ввиду, что необходимо уточнение этой формулы, включая суть и размерности входящих в нее величин. Естественным шагом является запись коэффициента теплопередачи с учётом термических сопротивлений стенки и слоя загрязнений для записи плотности теплового потока с стороны кипящего продукта, из этого следует:

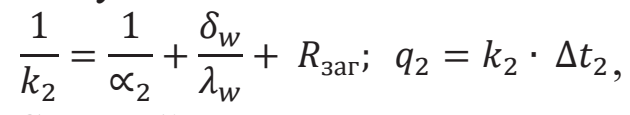

С другой стороны эта плотность теплового потока должна быть такой же, как и с стороны греющего пара, т.е.:

$q_{1}=\propto 1 \cdot \Delta t_{1} ; \Delta t_{1}=t_{g r}-t_{W 1}$

и $q_{1}=q_{2} ; t_{g r}-t_{W 1}=t_{v t}+\Delta t_{2}$.

Конкретные расчёты показали, что для каждого варианта температуры греющего пара, при всех остальных исходных условиях одинаковых, определение температуры поверхности кипения и плотности теплового потока, можно свести к решению квадратного уравнения вида:

$\mathrm{tW} 1^{2}-2 \cdot \mathrm{tW} 1 \cdot \operatorname{tgr} 11-\frac{\mathrm{Z} 111}{\mathrm{Z} 122^{2}} \cdot \mathrm{tW} 1+\mathrm{t} 10 \cdot \frac{\mathrm{Z} 111}{\mathrm{Z}_{12} 2^{2}}+\operatorname{tgr}^{2}{ }^{2}=\cdot 0 \cdot(22)$ Здесь: $t W 1 ; t_{g r} 11 ; t 10 ; Z 111 ; Z 122-$ искомая температура на поверхности кипения; температура греющего пара в этом варианте; температура насыщения вторичного пара; комплексы основных факторов влияния, включая связанные с теплофизическими свойствами продукта и теплоносителя и геометрией поверхностей теплообмена. Для каждого варианта температуры греющего пара определялись: температуры поверхности кипения, плотности теплового потока и масштаб поверхности парогенератора, а затем её масса, стоимость и прочие параметры для техно- экономического расчёта и определения оптимального значения греющей температуры. Результаты расчётов в их графической форме представлены ниже для разных вариантов исходных данных.

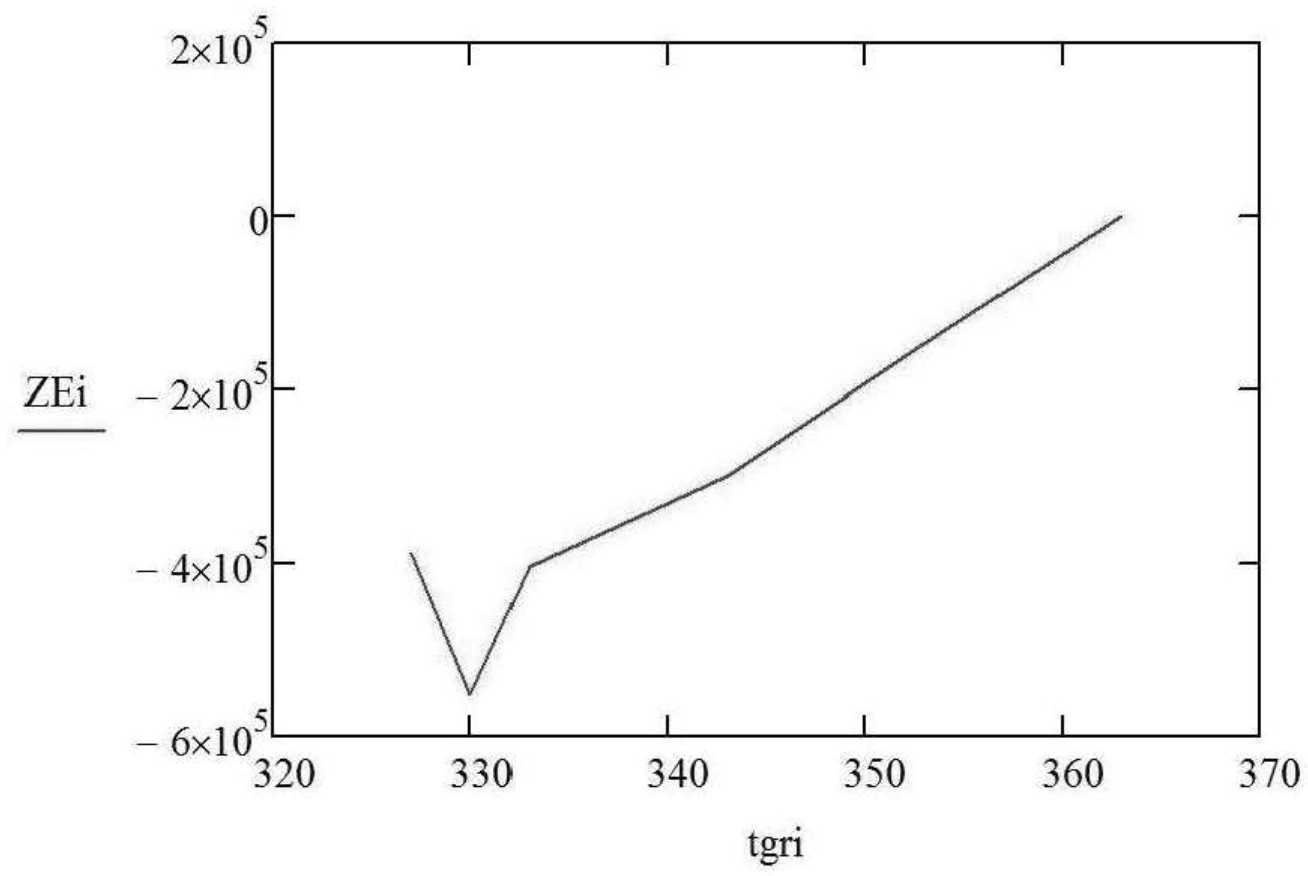

Рис. 1. Зависимость критерия оптимизации ZEі от греющей температуры $t_{\text {gri }}$ при 7500 часах в год работы и прочих вышеназванных параметрах. 


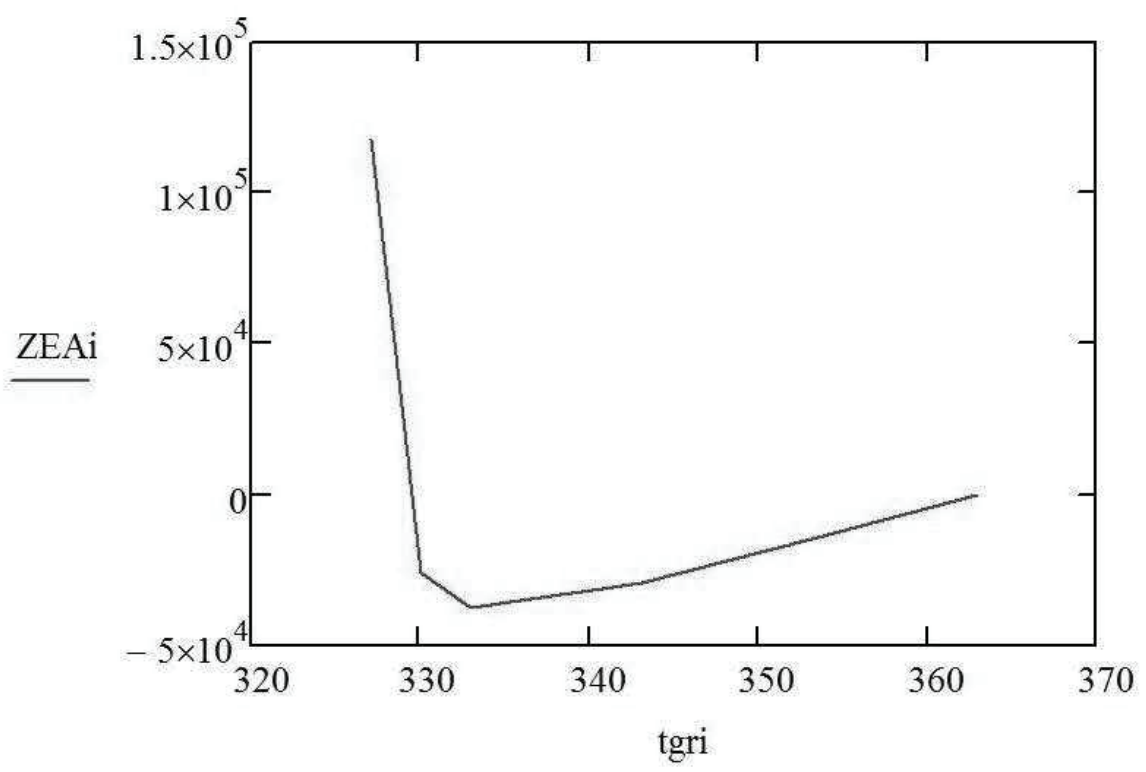

Pис. 2. Зависимость критерия оптимизации ZEAi от греющей температуры $t_{g r i}$ при 750 часах в год работы и прочих выщеназванных параметрах.

\section{Исходные положения по выбору оптималь- ных параметров ВВУ}

Задача оптимизации ВВУ с ТНУ состоит в определении режимных параметров ТНУ, таких как температуры испарения и конденсации хладагента, которые обеспечат наименьшие затраты энергии на проведение процесса при наименьших эксплуатационных затратах и соблюдении технологических ограничений. Следует также учесть влияние перегрева и переохлаждения хладагента на эффективность работы ТНУ.

Для решения задачи был принят критерий оптимизации в таком виде:

Копт $=$ Сэ·Зэ + Ка·Кк,

где Зэ - потребленная за год энергия; Ка - коэффициент амортизации Кк - стоимость оборудования.

Возможны варианты использования только электрической энергии либо комбинированное использование электрической и тепловой энергии. Во втором случае критерий примет следующий вид:

Копт =Сээ·Зээ + Стэ·Зтэ +Ка·Кк,

где: Сээ - стоимость электроэнергии; Зээ - потребленная за год электроэнергия; Стэ - стоимость тепловой энергии; Зтэ - потребленная за год тепловая энергия; Параметрами, влияющими на показатель качества (критерий оптимальности) являются температуры испарителя и конденсатора, температура перегрева хладагента, конструктивные особенности теплообменников (расположение труб, их длина или диаметр). Для оптимизации ВВУ с ТНУ принята математическая модель.

Алгоритм реализации модели включает следующие этапы:

1. Ввод исходных данных. На этом этапе задаются производительностью аппарата, вид сырья, его начальная температура, давление в аппарате, температура охладителя и конденсатора ТН, температура перегрева хладагента в ТН, расположение теплообменных труб и их линейные размеры. Стоимость электричества и тепла и коэффициент амортизации.

2. Определение теплофизических свойств продукта и хладагента.

3. Определение температуры кипения продукта в аппарате с учетом давления в аппарате и температурной депрессии.

4. Определение теплофизических свойств продукта и жидкого и парообразного растворителя при температуре кипения продукта.

5. Определение расхода тепла. По величине расхода тепла определяется мощность компрессора ТН и величина мощности подводимой к 
установке либо сбрасываемой в окружающую среду.

6. Определяется стоимость подводимой за год электрической и тепловой энергии.

7. Определяется коэффициент теплопередачи кипятильных труб ВВА, которые являются конденсатором ТНУ.

8. Определяется площадь поверхности кипятильных труб и их стоимость.

9. Определяется коэффициент теплопередачи конденсатора ВВА, который является испарителем ТНУ.

10. Определяется площадь поверхности конденсатора и его стоимость.

11. Определяются суммарные эксплуатационные затраты за год как сумма стоимости потребленной за год энергии и амортизационных отчислений от капитальных затрат на теплообменное оборудование.

\section{Выводы и рекомендации}

Таким образом, по результатам выполненных расчётов при принятом виде функции цели (критерии оптимальности) следует, что оптимальной является температура греющего пара при числе часов работы ВВУ в 7500 в год в 330 K, т.е. 57 C. В случае 750 часов работы в году, эта температура составляет примерно $333 \mathrm{~K}$, т.е. 60С. Отметим, что такой функционал и критерий оптимизации возникли для принятых исходных условий и исходных стоимостных параметров (стоимость единицы массы оборудования, в данном случае парогенератора; цена единицы энергии, которая может быть получена, если в качестве греющего пара используется пар из отбора турбины; стоимость единицы электрической энергии и т.д.). При использовании греющего пара из котельной, с точки зрения денежных затрат, не имеет значения вероятный энергетический потенциал этого пара. Тем не менее, при сравнении варианта с традиционной схемой подвода тепла к ВВУ и варианта, когда источником энергии для ВВУ является тепловой насос, нужной производительности или система на его основе, такой подход к выбору оптимальных температурных параметров парогенератора, представляется естественным.

\section{ЛИТЕРАТУРА}

1. Adriano V. Ensinas, Silvia A. Nebra, Miguel A. Lozano, Luis Serra. Design of Evaporation Systems and Heaters Networks in Sugar Cane Factories Using a Thermoeconomic Optimization Procedure. // Int. J. of Thermodynamics ISSN 1301-9724 Vol. 10 (No. 3), pp. 97-105, September 2007

2. Бурдо О.Г.Энергетический мониторинг пищевых производств - Одесса: Полиграф, 2008 244 c.

3. Бурдо О. Г., Буйвол С. М., Бандура В. Н. Энергетическая стратегия развития агропромышленного комплекса в условиях кризиса // Проблемы региональной энергетики. 2009, №1.

4. R. Simpson, S. Almonacid, D. Lopez, and A. Abakarov. "Optimum design and operating conditions of multiple effect evaporators: Tomato paste," J. Food Eng., 2008 - Vol. 89, P. 488-497.

5. M. H. Khademi, M. R. Rahimpour, and A. Jahanmiri. "Simulation and optimization of a sixeffect evaporator in a desalination process," Chem. Eng. Proc. - 2009, Vol. 48, P. 339-347.

6. Марченко В. Н., Прокопов М. Г. Энергетическая эффективность парового струйного термокомпрессора // Холодильна техніка і технологія. - 2007. - № 5 (109). - С. 45-51.

7. В. Арсеньєв, С. Шарапов, В. Мірошниченко Застосування рідинно-парового ежектора для рекомпресії вторинної пари вакуумних випарних установок // Энергосбережение. Энергетика. Энергоаудит.. - 2013. - № 2. - С. 57-64.

8. James Pope and Jeffrey Hude. Industrial performance of the mechanical vapor recompression and multiple effect evaporator system: successful operation and significant reduction in steam usage // American society of sugar beet technologists. Biennial meeting / Proceedings from the 31st Biennial Meeting February 28 - March 3, 2001, Vol. 31 P. 124-136.

9. K. J. Chua, S. K. Chou, and W. M. Yang. "Advances in heat pump systems: A review," Appl. Energy, 2010, Vol. 87, P. 3611-3624.

10. Chi-I Tuan, Yi-Lung Yeh, Ting-Chien Chen, Chi-Jen Chen. The Optimal Operation Design for a Three-effect Vacuum Evaporator (TEVE) and an Energy Performance Assessment. // International Journal of Energy Science IJES. -2011, Vol.1 №.2 P.118-124. 


\section{THE PROBLEM OF SELECTING THE BEST TECHNICAL SOLUTIONS OF HEAT SUPPLY FOR VACUUM EVAPORATOR}

\section{Smirnov H.F., Zykov A.V., Reznichenko D.N.}

Odessa National Academy of Food Technology (ONAFT); Kanatna Str. 112, Odessa; Ukraine

The principles of the Vacuum Evaporator (VE) optimal parameters determination were suggested. The problem was studied on the example of tomato juice concentration in VE. The special method for different variants of VE optimization was working out. It is especially important for the correct comparison of usual VE design (using steam from the boiler or from the turbine) and VE with heat pump as the energy source. The optimization calculations were completely fulfilled. The optimal steam temperature was discovered from these results. The dependency of minimum expenditures from the inlet and outlet concentrations was obtained. It was concluded, that there are some conditions, when VE with Heat Pump can be considerable better. The using of Heat Pump as the energy source for VE would be especially effective in the cases when temperature drop between heating and cooling temperatures is low. References:10, figures 2.

Key words: vacuum evaporator, heat pump, optimization.

1. Adriano V. Ensinas, Silvia A. Nebra, Miguel A. Lozano, Luis Serra. Design of Evaporation Systems and Heaters Networks in Sugar Cane Factories Using a Thermoeconomic Optimization Procedure. // Int. J. of Thermodynamics ISSN 1301-9724 Vol. 10 (No. 3), P. 97-105, September 2007.

2. Burdo O.G. Energy monitoring of food production - Odessa: Poligraf, 2008 - 244 p (Rus.)

3. Burdo O.G., Buyvol S.M., Bandura V.N. Energy Strategy of development of agriculture in the conditions of crisis // Problemy regionalnoy energetiki. 2009, №1 (Rus.)

4. R. Simpson, S. Almonacid, D. Lopez, and A. Abakarov. "Optimum design and operating conditions of multiple effect evaporators: Tomato paste," J. Food Eng.,2008, Vol. 89, P. 488-497.

5. M.H. Khademi, M.R. Rahimpour, and A. Jahanmiri. "Simulation and optimization of a sixeffect evaporator in a desalination process," Chem. Eng. Proc., 2009, Vol. 48, P. 339-347.

6. Marchenko V.N., Prokopov M.G. The energy efficiency of the steam jet thermocompressor // Holodylna tehnika i tehnologiya - 2007. - № 5 (109). - P. 45-51. (Rus.)

7. V. Arsenev, S. Shrapov, V. Miroshnichenko The using of liquid-vapor ejector for secondary vapor recompression in vacuum evaporators // Energosberezhenie. Energetika. Energoaudit. 2013. - N 2. - P. 57-64. (Ukr.)

8. James Pope and Jeffrey Hude. Industrial performance of the mechanical vapor recompression and multiple effect evaporator system: successful operation and significant reduction in steam usage // American society of sugar beet technologists. Biennial meeting / Proceedings from the 31st Biennial Meeting February 28 - March 3, 2001, Vol. 31, P. 124-136.

9. K. J. Chua, S. K. Chou, and W. M. Yang. "Advances in heat pump systems: A review," Appl. Energy, 2010, Vol. 87, P. 3611-3624.

10. Chi-I Tuan, Yi-Lung Yeh, Ting-Chien Chen, Chi-Jen Chen. The Optimal Operation Design for a Three-effect Vacuum Evaporator (TEVE) and an Energy Performance Assessment. // International Journal of Energy Science IJES. - 2011,Vol.1, No.2, P.118-124. 